\title{
THE STABILITY OF THE EXPONENTIAL EQUATION
}

\author{
ROMAN GER AND PETER ŠEMRL
}

(Communicated by J. Marshall Ash)

\begin{abstract}
We generalize the well-known Baker's superstability result for exponential mappings with values in the field of complex numbers to the case of an arbitrary commutative complex semisimple Banach algebra. It was shown by Ger that the superstability phenomenon disappears if we formulate the stability question for exponential complex-valued functions in a more natural way. We improve his result by showing that the maximal possible distance of an $\varepsilon$-approximately exponential function to the set of all exponential functions tends to zero as $\varepsilon$ tends to zero. In order to get this result we have to prove a stability theorem for real-valued functions additive modulo the set of all integers $\mathbb{Z}$.
\end{abstract}

\section{INTRODUCTION}

Let $(S,+)$ be an arbitrary semigroup, and let $f$ map $S$ into the field $\mathbf{C}$ of all complex numbers. Assume that $f$ is an approximately exponential function, i.e., there exists a nonnegative number $\varepsilon$ such that

$$
|f(x+y)-f(x) f(y)| \leq \varepsilon \quad \text { for } x, y \in S .
$$

Then $f$ is either bounded or exponential (see Baker [1], Baker, Lawrence, and Zorzitto [2], and Kuczma [6]). The same result is also true for approximately exponential mappings with values in a normed algebra with the property that the norm is multiplicative [1]. In the same paper Baker gives the following example to show that this result fails if the algebra does not have the multiplicative norm property. Let $\varepsilon>0$, choose $\delta>0$ so that $\left|\delta-\delta^{2}\right|=\varepsilon$, and let $f: \mathbf{C} \rightarrow \mathbf{C} \oplus \mathbf{C}$ be defined as

$$
f(\lambda)=\left(e^{\lambda}, \delta\right), \quad \lambda \in \mathbf{C} .
$$

Then, with the nonmultiplicative norm given by $\|(\lambda, \mu)\|=\max \{|\lambda|,|\mu|\}$, we have $\|f(\lambda+\mu)-f(\lambda) f(\mu)\|=\varepsilon$ for all complex $\lambda$ and $\mu, f$ is unbounded, but it is not true that $f(\lambda+\mu)=f(\lambda) f(\mu)$ for all complex $\lambda$ and $\mu$. In this counterexample the algebra $\mathcal{A}=\mathbf{C} \oplus \mathbf{C}$ can be decomposed as a direct sum of two ideals $\mathcal{A}=I_{1} \oplus I_{2}$, $I_{1}=\{(\lambda, 0): \lambda \in \mathbf{C}\}$ and $I_{2}=\{(0, \lambda): \lambda \in \mathbf{C}\}$. If we denote by $P_{1}$ and $P_{2}$ the projections corresponding to this direct sum decomposition, then the mapping $P_{1} f$ is exponential, while $P_{2} f$ is bounded. We will show that such behavior is typical for approximately exponential mappings with values in an arbitrary semisimple complex commutative Banach algebra.

Received by the editors February 1, 1994 and, in revised form, July 24, 1994

1991 Mathematics Subject Classification. Primary 39 B72.

Key words and phrases. Exponential functions, congruentialy additive functions, stability. 
In a situation where an approximate homomorphism must be a true homomorphism we say that the equation of homomorphism is superstable. In a recent paper [4] the first author showed that the superstability phenomenon is caused by the fact that problem (1) is ill-posed in a sense. Namely, posing problem (1) one disregards the natural group structure in $\mathbf{C}$ which in this case should obviously be the multiplicative group $(\mathbf{C} \backslash\{0\}, \cdot)$ all of nonzero complex numbers. So, it seems more natural to pose the problem in the following way:

$$
\left|\frac{f(x+y)}{f(x) f(y)}-1\right| \leq \varepsilon, \quad x, y \in S,
$$

for functions $f: S \rightarrow \mathbf{C} \backslash\{0\}$. This apparently diminishes the class of functions considered since we have eliminated their possible zero values. In the case that $(S,+)$ is a group it is easy to see that every solution of inequality (1) such that $0 \in f(S)$ has to be bounded. It was shown by Ger [4] that the restriction $f(x) \neq 0$ for all $x \in S$ is inessential also in the case that $(S,+)$ is an arbitrary semigroup. In the same paper he described the stability behaviour of approximate homomorphisms with values in the group $(\mathbf{C} \backslash\{0\}, \cdot)$ in the following way. Let $(S,+)$ be an amenable semigroup, and let $\varepsilon \in[0,1)$ be a given number. Assume that $f: S \rightarrow \mathbf{C} \backslash\{0\}$ satisfies (2). Then there exists a function $g: S \rightarrow \mathbf{C} \backslash\{0\}$ such that $g(x+y)=$ $g(x) g(y), x, y \in S$

$$
\left|\frac{g(x)}{f(x)}-1\right| \leq \frac{2-\varepsilon}{1-\varepsilon}, \quad x \in S
$$

and

$$
\left|\frac{f(x)}{g(x)}-1\right| \leq \frac{2-\varepsilon}{1-\varepsilon}, \quad x \in S .
$$

It is the aim of this paper to improve the estimation

$$
\frac{2-\varepsilon}{1-\varepsilon}
$$

in inequalities (3) and (4) by a better one which tends to zero as $\varepsilon$ tends to zero.

The main tool in the proof of our result is a stability result for real-valued functions that are additive modulo the set of all integers $\mathbf{Z}$. This result is of independent interest. In the next section we will prove an even more general result of this type.

\section{The STABILITy OF CONGRUENTIALY ADDITIVE MAPPINGS}

In this section we will need the following notation. Let $(X,+)$ be a group and let $T \subset X$. Then $T^{+}$denotes the set $T+T$, while $T^{-}=T-T$.

Theorem 2.1. Let $(S,+)$ be a cancellative Abelian semigroup, and let $(X,+)$ be a torsion-free divisible Abelian group. Assume that $U$ and $V$ are nonempty subsets of $X$ satisfying

$$
\left(U^{+}\right)^{-} \cap\left(V^{+}\right)^{-}=\{0\} .
$$

Then each function $\alpha: S \rightarrow X$ with the property

$$
\alpha(x+y)-\alpha(x)-\alpha(y) \in U+V, \quad x, y \in S,
$$


admits a representation of the form $\alpha=\beta+\gamma$ where $\beta, \gamma: S \rightarrow X$ satisfy the relations

$$
\beta(x+y)-\beta(x)-\beta(y) \in U, \quad x, y \in S,
$$

and

$$
\gamma(x+y)-\gamma(x)-\gamma(y) \in V, \quad x, y \in S .
$$

The functions $\beta$ and $\gamma$ are determined uniquely up to an additive function.

Proof. There exist functions $\psi: S \times S \rightarrow U$ and $\varphi: S \times S \rightarrow V$ such that

$$
d(x, y)=\alpha(x+y)-\alpha(x)-\alpha(y)=\psi(x, y)+\varphi(x, y), \quad x, y \in S .
$$

The commutativity of $(S,+)$ implies that $d$ is symmetric. We will show that the same is true for function $\psi$. We have

$$
\psi(x, y)-\psi(y, x)=d(x, y)-\varphi(x, y)-d(y, x)+\varphi(y, x) \in V^{-} .
$$

On the other hand,

$$
\psi(x, y)-\psi(y, x) \in U^{-},
$$

and since $0 \in U^{-} \cap V^{-}$, we infer that

$$
\psi(x, y)-\psi(y, x) \in\left(U^{-}+U^{-}\right) \cap\left(V^{-}+V^{-}\right)=\left(U^{+}\right)^{-} \cap\left(V^{+}\right)^{-}=\{0\} .
$$

Our next step will be to prove that $\psi$ satisfies

$$
\psi(x, y+z)+\psi(y, z)=\psi(x+y, z)+\psi(x, y)
$$

for all $x, y \in S$. A straightforward computation yields that $d$ satisfies the same functional equation. Consequently,

$$
\begin{aligned}
& \psi(x, y+z)+\psi(y, z)-\psi(x+y, z)-\psi(x, y) \\
& \quad=\varphi(x+y, z)+\varphi(x, y)-\varphi(x, y+z)-\varphi(y, z) \\
& \quad \in\left(U^{+}\right)^{-} \cap\left(V^{+}\right)^{-}=\{0\},
\end{aligned}
$$

which proves (5). According to Hosszú theorem [5] there exists a function $\beta: S \rightarrow X$ such that

$$
\psi(x, y)=\beta(x+y)-\beta(x)-\beta(y) \in U, \quad x, y \in S .
$$

Let $\gamma=\alpha-\beta$. Then

$$
V \ni \varphi(x, y)=d(x, y)-\psi(x, y)=\gamma(x+y)-\gamma(x)-\gamma(y)
$$

for all $x, y \in S$, which completes the proof of the existence.

In order to prove the uniqueness we assume that we have two representations

$$
\alpha=\beta+\gamma=\beta_{1}+\gamma_{1}
$$

with the properties described above. Putting

$$
c=\beta-\beta_{1}=\gamma_{1}-\gamma
$$

we get

$$
\begin{aligned}
c(x+y)-c(x)-c(y)= & (\beta(x+y)-\beta(x)-\beta(y)) \\
& -\left(\beta_{1}(x+y)-\beta_{1}(x)-\beta_{1}(y)\right) \\
\in & U-U=U^{-} .
\end{aligned}
$$


Similarly,

$$
c(x+y)-c(x)-c(y) \in V^{-} .
$$

These two relations together with $0 \in U^{-} \cap V^{-}$imply the additivity of $c$. Moreover, we have

$$
\gamma_{1}=\gamma+c \text { and } \beta_{1}=\beta-c
$$

which completes the proof.

Theorem 2.2. Let $(S,+)$ be a cancellative Abelian semigroup, and let $X$ be a Banach space. Assume that nonempty subsets $U, V \in X$ satisfy $\left(U^{+}\right)^{-} \cap\left(V^{+}\right)^{-}=$ $\{0\}, 0 \in V$, and $V$ is bounded. Then for every function $\alpha: S \rightarrow X$ satisfying

$$
\alpha(x+y)-\alpha(x)-\alpha(y) \in U+V, \quad x, y \in S,
$$

there exists a function $p: S \rightarrow X$ such that

$$
p(x+y)-p(x)-p(y) \in U, \quad x, y \in S,
$$

and

$$
p(x)-\alpha(x) \in \operatorname{clconv} V=: V_{0}, \quad x \in S .
$$

The function $p$ is unique provided that $U^{-} \cap 3 V_{0}^{-}=\{0\}$.

Proof. According to the previous theorem we get the existence of functions $\beta, \gamma$ : $S \rightarrow X$ such that $\alpha=\beta+\gamma$,

$$
\beta(x+y)-\beta(x)-\beta(y) \in U, \quad x, y \in S,
$$

and

$$
\gamma(x+y)-\gamma(x)-\gamma(y) \in V, \quad x, y \in S .
$$

It follows from the Forti's version [3] of the Hyers-Ulam stability theorem for additive mappings that there exists an additive function $\delta: S \rightarrow X$ such that

$$
\delta(x)-\gamma(x) \in V_{0}, \quad x \in S .
$$

Putting $p=\delta+\beta$ and applying $-\gamma=\beta-\alpha$ we get the desired relation

$$
p(x)-\alpha(x) \in V_{0}, \quad x \in S .
$$

Next, we shall prove the uniqueness of $p$. Let $p_{1}, p_{2}: S \rightarrow X$ be two functions such that

$$
p_{i}(x+y)-p_{i}(x)-p_{i}(y) \in U, \quad x, y \in S,
$$

and

$$
p_{i}(x)-\alpha(x) \in V_{0}, \quad x \in S,
$$

$i=1,2$. Then $r(x)=p_{1}(x)-p_{2}(x)=\left(p_{1}(x)-\alpha(x)\right)-\left(p_{2}(x)-\alpha(x)\right) \in V_{0}^{-}$for all $x \in S$, and consequently

$$
r(x+y)-r(x)-r(y) \in(U-U) \cap\left(V_{0}^{-}-V_{0}^{-}-V_{0}^{-}\right)=U^{-} \cap 3 V_{0}^{-}=\{0\} .
$$

Here, we have used the fact that $V_{0}^{-}$is convex and symmetric with respect to zero. Thus, $r$ is additive and bounded which implies that $p_{1}-p_{2}=r=0$. This completes the proof. 
Corollary 2.3. Let $(S,+)$ be a cancellative Abelian semigroup, and let $X$ be a Banach space. Given an additive subgroup $(U,+)$ of $(X,+)$ and a bounded convex and symmetric with respect to zero subset $V$ of $X$ such that

$$
U \cap 4 V=\{0\},
$$

assume that a function $\alpha: S \rightarrow X$ satisfies the congruence

$$
\alpha(x+y)-\alpha(x)-\alpha(y) \in U+V, \quad x, y \in S .
$$

Then there exists a function $p: S \rightarrow X$ such that

$$
p(x+y)-p(x)-p(y) \in U, \quad x, y \in S,
$$

and

$$
\alpha(x)-p(x) \in \operatorname{cl} V, \quad x \in S .
$$

We will conclude this section by formulating an easy consequence of the previous results that will be needed in the sequel.

Corollary 2.4. Let $(S,+)$ be a cancellative Abelian semigroup, and let $\varepsilon \in\left(0, \frac{1}{4}\right)$. Assume that a mapping a from $S$ into the field of real numbers $\mathbf{R}$ satisfies the congruence

$$
\alpha(x+y)-\alpha(x)-\alpha(y) \in \mathbf{Z}+(-\varepsilon, \varepsilon), \quad x, y \in S .
$$

Then there exists a function $p: S \rightarrow \mathbf{R}$ such that

$$
p(x+y)-p(x)-p(y) \in \mathbf{Z}, \quad x, y \in S,
$$

and

$$
|\alpha(x)-p(x)| \leq \varepsilon, \quad x \in S
$$

\section{The STABILITY OF EXPONENTIAL MAPPINGS}

We will start this section by proving a result which extends the well-known Baker's superstability theorem for exponential mappings with values in the field of complex numbers to the case of an arbitrary commutative semisimple complex Banach algebra. Thus, we will overcome the "old" difficulty caused by the lack of multiplicativity of the norm once we leave the field $\mathbf{C}$.

Every commutative complex semisimple Banach algebra without unit can be isometrically embedded into one with unit. So, the presence of a unit will be assumed without special mention.

Our first result shows that Baker's superstability theorem can be extended to the case where the target space is a commutative semisimple complex Banach algebra under an additional unboundedness assumption.

Theorem 3.1. Let $(S,+)$ be a semigroup, and let $\mathcal{A}$ be a commutative semisimple complex Banach algebra. Assume that the mapping $f: S \rightarrow \mathcal{A}$ is such that

(a) the transformation $S^{2} \ni(x, y) \mapsto f(x+y)-f(x) f(y) \in \mathcal{A}$ is norm bounded,

(b) for every nonzero linear multiplicative functional $\varphi$ on $\mathcal{A}$ the set $(\varphi \circ f)(S)$ is unbounded.

Then $f$ is exponential. 
Proof. Put $F(x, y)=f(x+y)-f(x) f(y), x, y \in S$, and fix arbitrarily a member $\varphi$ of the set $\mathcal{M}(\mathcal{A})$ of all nonzero linear multiplicative functionals on $\mathcal{A}$. By the assumption, there exists an $\varepsilon>0$ such that $\|F(x, y)\| \leq \varepsilon$ for all $(x, y) \in S^{2}$. Hence

$$
\begin{aligned}
& |(\varphi \circ f)(x+y)-(\varphi \circ f)(x)(\varphi \circ f)(y)| \\
& \quad=|\varphi(F(x, y))| \leq\|\varphi\|\|F(x, y)\| \leq \varepsilon
\end{aligned}
$$

for all $(x, y) \in S^{2}$, because $\|\varphi\|=1$. Since $(\varphi \circ f)(S)$ is unbounded, an appeal to Baker's theorem [1] shows that $\varphi \circ f$ is an exponential functional, i.e., $F(x, y) \in$ $\operatorname{Ker} \varphi$ for all $(x, y) \in S^{2}$. Thus, for every $(x, y) \in S^{2}$, one has

$$
F(x, y) \in \bigcap\{\operatorname{Ker} \varphi: \varphi \in \mathcal{M}(\mathcal{A})\}=\operatorname{rad} \mathcal{A}=\{0\},
$$

since $\mathcal{A}$ is assumed to be semisimple. This proves that $f(x+y)=f(x) f(y)$ for all $x, y \in S$, which completes the proof.

In the next two theorems we will get rid of unboundedness assumption (b).

Theorem 3.2. Let $(S,+)$ be a semigroup and $\mathcal{A}$ a commutative $C^{*}$-algebra. Assume that $\varepsilon>0$ and that a mapping $f: S \rightarrow \mathcal{A}$ satisfies

$$
\|f(x+y)-f(x) f(y)\| \leq \varepsilon
$$

for all $x, y \in S$. Then there exists a commutative $C^{*}$-algebra $\mathcal{B}$ such that

(i) $\mathcal{A}$ is a $C^{*}$-subalgebra of $\mathcal{B}$ (and therefore, $f$ may be considered as a mapping from $S$ into $\mathcal{B})$,

(ii) algebra $\mathcal{B}$ can be represented as a direct sum $\mathcal{B}=I \oplus J$ where $I$ and $J$ are closed ideals,

(iii) if $P$ and $Q$ are projections corresponding to the direct sum decomposition $\mathcal{B}=I \oplus J$, then $P f$ is an exponential mapping and $Q f$ is norm-bounded.

Proof. Let us denote by $\Phi: \mathcal{A} \rightarrow C(\Delta)$ the Gelfand transform of $\mathcal{A}$ onto the algebra of all complex continuous functions on a compact Hausdorff space $\Delta$. It is easy to verify that for every $h \in \Delta$ the complex-valued function

$$
x \mapsto(\Phi(f(x)))(h), \quad x \in S,
$$

is $\varepsilon$-approximately exponential. It follows from Baker's result [1] that it is exponential or

$$
|(\Phi(f(x)))(h)| \leq \frac{1+\sqrt{1+4 \varepsilon}}{2}=: \delta \quad \text { for all } x \in S .
$$

We denote by $\Delta^{2}$ the subset of all points $h$ from $\Delta$ satisfying (6). For every $x \in S$ we denote by $\Delta_{x}$ the subset of all points $h$ from $\Delta$ satisfying $|(\Phi(f(x)))(h)| \leq \delta$. Obviously,

$$
\Delta^{2}=\bigcap_{x \in S} \Delta_{x}
$$

and consequently, $\Delta^{2}$ is a closed subset of $\Delta$. Set $\Delta^{1}=\operatorname{cl}\left(\Delta \backslash \Delta^{2}\right)$. It is easy to see that

$$
(\Phi(f(x+y)))(h)=(\Phi(f(x)))(h)(\Phi(f(y)))(h), \quad x, y \in S,
$$

holds for all $h \in \Delta^{1}$. 
Let us define a commutative $C^{*}$-algebra $\mathcal{B}$ by $\mathcal{B}=C\left(\Delta^{1}\right) \oplus C\left(\Delta^{2}\right)$ with norm $\|\tau \oplus v\|=\max \{\|\tau\|,\|v\|\}$ for every $\tau \oplus v \in \mathcal{B}$. A mapping

$$
\tau \mapsto \tau_{\mid \Delta^{1}} \oplus \tau_{\mid \Delta^{2}}
$$

is an isometric $*$-homomorphism of $C(\Delta)$ into $\mathcal{B}$. Thus, $\mathcal{A}$ can be regarded as a $C^{*}$-subalgebra of $\mathcal{B}$. We denote $I=C\left(\Delta^{1}\right) \oplus\{0\}$ and $J=\{0\} \oplus C\left(\Delta^{2}\right)$. One can now easily see using (6) and (7) that (iii) is satisfied. This completes the proof.

The same approach can be applied when the target space of an approximately exponential mapping is a semisimple commutative Banach algebra. However, in this case the Gelfand transform need not be an isometry. More precisely, we have $\rho(f(x))=\|\Phi(f(x))\|, x \in S$, where $\rho$ denotes the spectral radius. Thus, we have the following result.

Theorem 3.3. Let $(S,+)$ be a semigroup and $\mathcal{A}$ a commutative semisimple complex Banach algebra. Assume that $\varepsilon>0$ and that a mapping $f: S \rightarrow \mathcal{A}$ satisfies

$$
\|f(x+y)-f(x) f(y)\| \leq \varepsilon
$$

for all $x, y \in S$. Then there exists a commutative $C^{*}$-algebra $\mathcal{B}$ such that

(i) there exists a continuous monomorphism $\Lambda$ of $\mathcal{A}$ into $\mathcal{B}$ with $\rho(a)=\|\Lambda(a)\|$, $a \in \mathcal{A}$,

(ii) algebra $\mathcal{B}$ can be represented as a direct sum $\mathcal{B}=I \oplus J$ where $I$ and $J$ are closed ideals,

(iii) if $P$ and $Q$ are projections corresponding to the direct sum decomposition $\mathcal{B}=I \oplus J$, then $P \Lambda f$ is an exponential mapping and $Q \Lambda f$ is norm-bounded.

From now on we are going to deal with the stability question formulated in (2). Using the results from the previous section we will improve Ger's [4] stability theorem for complex-valued exponential functions.

Theorem 3.4. Let $(S,+)$ be a cancellative Abelian semigroup, and let $\varepsilon \in[0,1)$ be a given number. Assume that $f: S \rightarrow \mathbf{C} \backslash\{0\}$ satisfies (2). Then there exists a unique function $g: S \rightarrow \mathbf{C} \backslash\{0\}$ such that $g(x+y)=g(x) g(y), x, y \in S$,

$$
\left|\frac{f(x)}{g(x)}-1\right| \leq \sqrt{1+\frac{1}{(1-\varepsilon)^{2}}-2 \sqrt{\frac{1+\varepsilon}{1-\varepsilon}}}, \quad x \in S,
$$

and

$$
\left|\frac{g(x)}{f(x)}-1\right| \leq \sqrt{1+\frac{1}{(1-\varepsilon)^{2}}-2 \sqrt{\frac{1+\varepsilon}{1-\varepsilon}}}, \quad x \in S .
$$

Remark. It was shown by Ger [4] that the assumption $\varepsilon<1$ is indispensible in the above stability result.

Proof. Every nonzero complex number $\lambda$ can be uniquely written as

$$
\lambda=|\lambda| \exp (i \arg (\lambda)),
$$

where $-\pi<\arg (\lambda) \leq \pi$. Then (2) implies

$$
\left|\frac{|f(x+y)|}{|f(x)||f(y)|} \exp (i(\arg (f(x+y))-\arg (f(x))-\arg (f(y))))-1\right| \leq \varepsilon
$$


for all $x, y \in S$. It follows that

$$
1-\varepsilon \leq \frac{|f(x+y)|}{|f(x)||f(y)|} \leq 1+\varepsilon
$$

and

$$
\arg (f(x+y))-\arg (f(x))-\arg (f(y)) \in 2 \pi \mathbf{Z}+[-\arcsin \varepsilon, \arcsin \varepsilon]
$$

for all $x, y \in S$. As $\varepsilon<1$ we have necessarily that $\arcsin \varepsilon<\pi / 2$. This inequality together with Corollary 2.4 imply the existence of a function $p: S \rightarrow \mathbf{R}$ such that

$$
p(x+y)-p(x)-p(y) \in 2 \pi \mathbf{Z}, \quad x, y \in S,
$$

and

$$
|p(x)-\arg (f(x))| \leq \arcsin \varepsilon, \quad x \in S .
$$

Put $h(x)=|f(x)|$. Then (10) yields

$$
1-\varepsilon \leq \frac{h(x+y)}{h(x) h(y)} \leq 1+\varepsilon
$$

for all $x, y \in S$. Consequently,

$$
|\ln h(x+y)-\ln h(x)-\ln h(y)| \leq-\ln (1-\varepsilon), \quad x, y \in S .
$$

Thus, in view of Rätz's result [7], there exists a homomorphism $q$ from $(S,+)$ into the additive group $(\mathbf{R},+)$ of all real numbers such that

$$
|q(x)-\ln h(x)| \leq-\ln (1-\varepsilon), \quad x \in S .
$$

We define a function $g: S \rightarrow \mathbf{C} \backslash\{0\}$ by $g(x)=\exp (q(x)+i p(x))$. It follows from the additivity of $q$ and (11) that $g$ is an exponential function. Furthermore, for every $x \in S$ we have

$$
\left|\frac{f(x)}{g(x)}-1\right|=|\exp (\ln h(x)-q(x)) \exp (i(\arg (f(x))-p(x)))-1| .
$$

Applying (12) and (13) we see that the complex number $f(x) / g(x)$ belongs to the set

$$
\Omega=\left\{\lambda \in \mathbf{C}: 1-\varepsilon \leq|\lambda| \leq(1-\varepsilon)^{-1} \text { and }-\arcsin \varepsilon \leq \arg (\lambda) \leq \arcsin \varepsilon\right\} .
$$

Obviously,

$$
\begin{aligned}
\sup \{|\lambda-1|: \lambda \in \Omega\} & =\left|(1-\varepsilon)^{-1} \exp (i \arcsin \varepsilon)-1\right| \\
& =\sqrt{1+\frac{1}{(1-\varepsilon)^{2}}-2 \sqrt{\frac{1+\varepsilon}{1-\varepsilon}}}
\end{aligned}
$$

which proves (8). The proof of (9) goes through in exactly the same way.

\section{ACKNOWLEDGMENT}

The second author wishes to express his gratitude to the organizers for inviting him to the Fourth International Conference on Functional Equations and Inequalities, Krynica, Poland, February 1993, during which time this work was completed. This research was supported in part by KBN grant No. 2 P03A 04909 (Poland) and by a grant from the Ministry of Science of Slovenia. 


\section{REFERENCES}

1. J. A. Baker, The stability of the cosine equation, Proc. Amer. Math. Soc. 80 (1980), 411-416. MR 81m:39015

2. J. A. Baker, J. Lawrence, and F. Zorzitto, The stability of the equation $f(x+y)=f(x) f(y)$, Proc. Amer. Math. Soc. 74 (1979), 242-246. MR 80d:39009

3. G. L. Forti, The stability of homomorphism and amenability, with applications to functional equations, Abh. Math. Sem. Univ. Hamburg 57 (1987), 215-226. MR 89b:39013

4. R. Ger, Superstability is not natural, Rocznik Naukowo-Dydaktyczny WSP w Krakowie, Prace Mat. 159 (1993), 109-123. CMP 95:09

5. M. Hosszú, On the functional equation $F(x+y, z)+F(x, y)=F(x, y+z)+F(y, z)$, Period. Math. Hungar. 1 (1971), 213-216. MR 44:7176

6. M. Kuczma, An introduction to the theory of functional equations and inequalities, PWN, Warszawa, Kraków, and Katowice, 1985. MR 86i:39008

7. J. Rätz, On approximately additive mappings, General Inequalities 2, Birkhäuser Verlag, Basel, Boston, and Stuttgart, 1980, pp. 233-251. MR 83e:90131

Institute of Mathematics, Silesian University, Bankowa 14, 40-007 Katowice, Poland

TF, University of Maribor, Smetanova 17, P.O. BOX 224, 62000 Maribor, Slovenia 